\title{
Classification of weed in soybean crops using unmanned aerial vehicle images
}

\author{
$1^{\text {st }}$ Nícolas Alessandro de Souza Belete \\ Universidade Federal de Rondônia \\ Universidade Católica Dom Bosco \\ Cacoal/RO, Campo Grande/MS, Brasil \\ nicolas.belete@unir.br
}

\author{
$2^{\text {nd }}$ Everton Castelão Tetila \\ Universidade Federal da Grande Dourados \\ Universidade Católica Dom Bosco \\ Dourados/MS, Campo Grande/MS, Brasil \\ evetontetila@ufgd.edu.br
}

\author{
$3^{\text {rd }}$ Gilberto Astolfi \\ Instituto Federal de Mato Grosso do Sul \\ Universidade Federal de Mato Grosso do Sul \\ Campo Grande, MS, Brasil \\ gilberto.astolfi@ifms.edu.br
}

\author{
$4^{\text {th }}$ Hemerson Pistori \\ Universidade Católica Dom Bosco \\ Universidade Federal de Mato Grosso do Sul \\ Campo Grande, MS, Brasil \\ pistori@ucdb.br
}

Resumo-Soybeans have been Brazil's main agricultural commodity, contributing substantially to the country's trade balance. However, their production and productivity costs are affected by weeds, diseases and pests. This paper proposes a computer vision system to monitor weeds in soybean fields using images captured by a UAV. The proposed system adopts the SLIC superpixels segmentation method to detect the plants in the images and visual attributes to describe the characteristics of the physical properties of the leaf, such as color, gradient, texture and shape. Our methodology evaluated the performance of three classifiers ( $k$ NN, RandonForest and SVM) for images captured at a height of 3 meters. The best results were obtained by the SVM classification algorithm with accuracy of $91.34 \%$. However, the results do not yet indicate that our approach can support experts and farmers in weed monitoring in soybean crops, requiring more images and experiments.

Index Terms-classification, weeds, soybean crop, UAV, images

\section{INTRODUCTION}

As plantas daninhas, também chamadas de ervas daninhas ou plantas invasoras, são conceituadas como plantas indesejadas que crescem em ambientes agrícolas de forma espontânea, competindo diretamente pelos mesmos recursos que as culturas plantadas necessitam para seu desenvolvimento. Portanto, dependendo do nível de infestação das plantas daninhas, a plantação pode sofrer prejuízos como diminuição da qualidade e produtividade [1].

Rizzardi e Fleck [2] trazem como possíveis prejuízos para a lavoura a perda de qualidade pela manifestação de pragas, doenças e impurezas decorrentes da colheita. Os autores complementam que a diminuição da produtividade nas lavouras é decorrente da competitividade das plantas por luz, água, nutrientes e espaço, aumentam os custos de produção e diminuindo o valor da lavoura.

Diante disso, a visão computacional surge como uma alternativa para auxiliar os agricultores no gerenciamento de suas plantações, pois, esta área pode automatizar tarefas, onde sensores óticos realizam um papel importante, como detecção de pragas e doenças nas lavouras, análise de comportamento animal, orientação de máquinas, silvicultura e outros [3].

Aplicações de sensoriamento remoto com imagend de veículos aéreos não tripulados (VANTs) aumentaram as oportunidades de proteção mais precisa das culturas, incluindo benefícios econômicos (redução de custos) e ambientais (redução do risco de poluição) [4].

Estudos recentes sobre proteção de culturas, usando imagens obtidas por VANTs e algoritmos de detecção remota baseados em aprendizado de máquina, foram propostos por Garcia-Ruiz [5] na identificação de doenças em cítricos, Torres-Sanchez et al. [6] [7] no mapeamento de plantas daninhas em lavouras de trigo. Peña et al. [8], Pérez-Ortiz et al. [9] [10]; LópezGranados [11]; e Castaldi et al. [12], apresentaram propostas para o monitoramento de plantas daninhas em lavouras de girassol e milho.

Portanto, tendo a visão computacional e os métodos de aprendizado de máquinas desempenhado um papel importante para a medição automática e classificação das imagens remotas, este trabalho propõe um sistema de visão computacional para identificar automaticamente plantas daninhas, por espécie, em lavouras de soja, a partir de imagens aéreas capturadas por veículos aéreos não tripulados (VANT).

\section{ABORDAGEM PROPOSTA}

A abordagem proposta (Figura 1) é um sistema de visão computacional para classificar, por espécie, plantas daninhas em lavouras de soja, utilizando imagens capturadas por VANT. Esta abordagem possui cinco etapas: (a) aquisição de imagens, (b) segmentação, (c) criação do banco de imagens, (d) extração de atributos e (e) classificação.

A primeira etapa consiste na captura das imagens de plantações de soja, utilizando um veículo aéreo não tripulado. A segunda etapa é anotação e segmentação das plantas daninhas presentes nas imagens, realizadas com os softwares LabelMe 


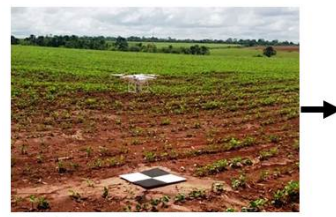

(a) Aquisição de Imagens

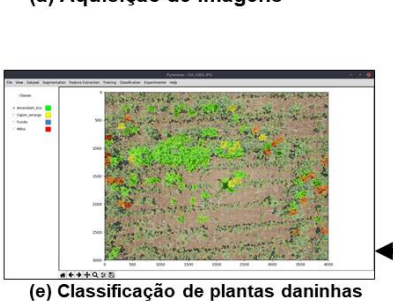

(e) Classificação de plantas daninhas

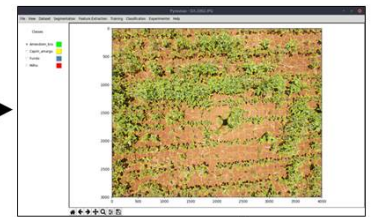

(b) Segmentação SLIC

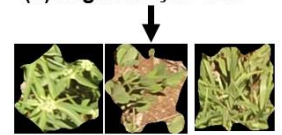

(c) Conjunto de imagens

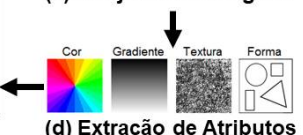

Figura 1. Proposta do sistema de visão computacional para classificar, por espécie, plantas daninhas em lavouras de soja, utilizando imagens capturadas por VANT.

e o Pynovisão. A terceira etapa consiste na a criação de um banco de imagens rotulado para treinamento dos algorítimos.

A quarta etapa consiste na extração de vetores de atributos dos segmentos do banco de superpixels, utilizando uma coleção de algoritmos extratores de cor, forma, gradiente e textura. Na ultima etapa é realizado o treinamento e teste dos algoritmos de aprendizagem supervisionadas selecionados, a partir dos dados extraídos na forma de vetor de atributos na etapa anterior. Em seguida, uma imagem é submetida aos processos de segmentação e classificação retornando à presença ou não de plantas daninhas (Figura 2).

\section{MATERIAIS E METODOS}

Para realização deste trabalho, a coleta de imagens foi realizada em uma área experimental com cultivares de soja convencional e ausência de aplicação de herbicidas. Esta área está situada na fazenda experimental da Universidade Federal da Grande Dourados (UFGD), localizada no município de Dourados-MS, Brasil, com coordenadas geográficas 22 13'57.52" latitude Sul e 5459'17.93” longitude Oeste.

$\mathrm{Na}$ etapa de captura de imagens foram obtidas 15 imagens da plantação de soja, a 3 metros de altura, no período diurno, durante a safra 2017/2018. Cada imagem tem uma dimensão de $4.000 \times 3.000$ pixels, totalizando 12 megapixels. Estas imagens foram capturadas com o veículo aéreo DJI Phanton 3 Professional, equipado com um sensor Sony EXMOR de 1/2,3 polegadas e 12 megapixels de resolução. A Figura 3 apresenta um exemplo das imagens obtidas na aquisição.

Para realização das próximas etapas, utilizamos o software Pynovisão, desenvolvido pelo Grupo de Pesquisa INOVISÃO - UCDB, e um notebook com processador Core i7 e memória RAM de $8 \mathrm{~GB}$.

$\mathrm{Na}$ etapa de anotação das plantas daninhas presentes nas imagens, 3 imagens selecionadas aleatoriamente foram submetidas a anotação com o software LabelMe que permite construir uma imagem com rótulos a serem usados para pesquisa de detecção e reconhecimento de objetos (Figura 3).

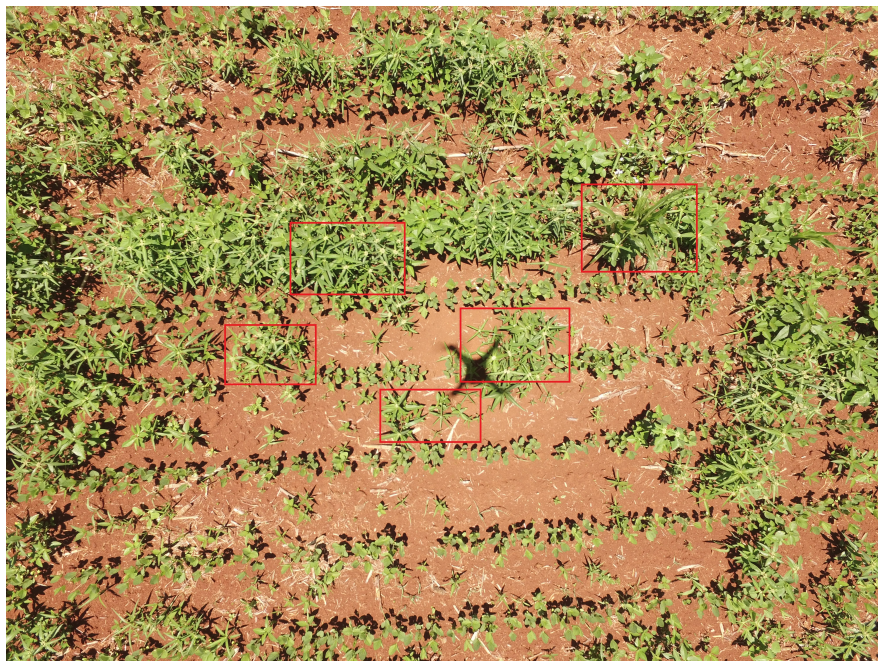

Figura 2. Imagem da plantação de soja com presença de plantas daninhas, capturada pelo VANT a $3 \mathrm{~m}$ de altura

Cabe ressaltar que cada imagem tinha prevalência de apenas uma espécie de planta daninha.

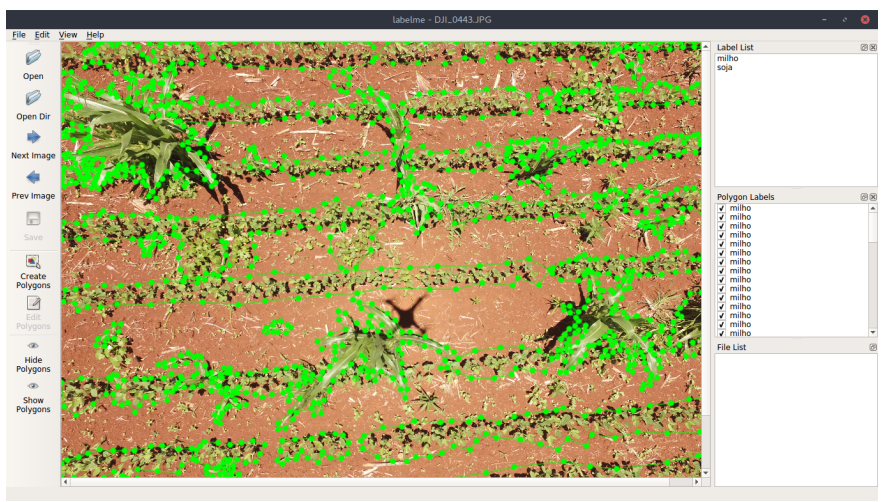

Figura 3. Tela do software LabelMe com uma imagem anotada com as classes milho e soja

Na etapa de segmentação optamos pela adoção do algoritmo Simple Linear Iterative Clustering (SLIC) superpixels. Este método emprega o algoritmo k-means para a geração de regiões semelhantes, chamadas superpixels [13]. Por padrão, antes do processo de segmentação, os parâmetros de entrada que o usuário pode definir são: a) o número de superpixels que deseja formar na imagem, determinado pelo parâmetro k; b) o valor da compacidade do segmento, permitindo definir a forma do superpixel; e c) o valor de suavização da imagem, determinado pelo parâmetro sigma.

Para este experimento definimos um valor $\mathrm{k}=1000$, compacidade $=35$ e sigma $=5$. Para definição destes valores vários testes foram realizados buscando uma combinação que permitisse obter um segmento com características predominantes a uma das classes definidas no estudo.

Como resultado da etapa de segmentação e anotação do banco de imagens para treinamento e teste foram obtidas 4 (quatro) pastas com 134, 189, 82 e 2.355 imagens para as 
classes Amendoim Bravo (Euphorbia heterophylla), Capim Amargoso (Digitaria insularis), Milho (Zea mays L.) e Fundo, respectivamente. A Figura 4 exemplifica a segmentação realizada pelo software Pynovisão, onde é possível perceber como os superpixels foram formados e a que nível de detalhes (características).

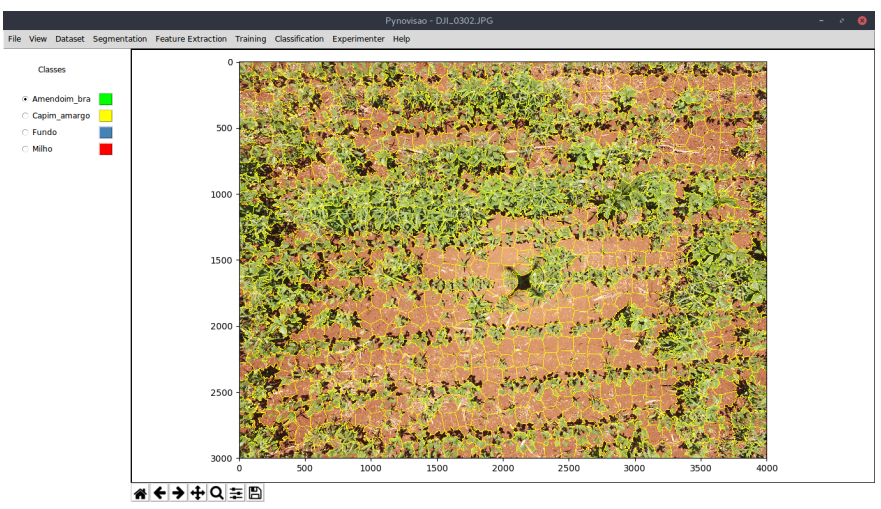

Figura 4. Imagem segmentada com o algoritmo SLIC superpixels no software Pynovisão.

Após a etapa da segmentação, cada superpixel foi anotado automaticamente, a partir da imagem anotada com o software LabelMe, entre as classes Amendoim Bravo, Capim Amargoso, Milho e Fundo, formando o banco de imagens para treinamento e teste do sistema proposto (Figura 5).
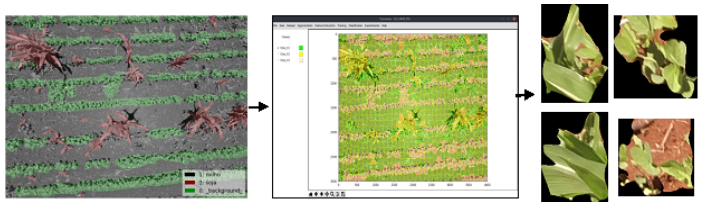

Figura 5. Da esquerda para a direita temos a imagem anotada com o software LabelMe, os segmentos de superpixels anotados automaticamente e exemplos de superpixels do banco de imagens

Após a etapa de criação do banco de imagens, realizamos a etapa de extração de atributos de cada segmento de superpixel, utilizando os extratores: estatística de cores [14] para cor; matriz de co-ocorrência de níveis de cinza [15] e padrões binários locais [16] para textura; histograma de gradientes orientados [17] para forma e orientação; Momento de $\mathrm{Hu}$ e momentos centrais [18] para momento invariante de escala e rotação. Este grupo de extratores foi composto por 218 atributos. O resultado desta etapa foi um arquivo no padrão ARFF (Attribute-Relation File Format), contendo vetores de atributos extraídos das imagens de superpixels com vários atributos visuais que descrevem as propriedades físicas das imagens: cor, gradiente, textura e forma.

Para a classificação das imagens empregamos três algoritmos de aprendizagem supervisionada bem conhecidos na literatura, sendo eles: Floresta Aleatória (Random Forest) [19], k-vizinhos mais próximos (k-NN) [20], e maquina de vetores de suporte (SMV) [21]. Os experimentos mantendo a configuração padrão dos algorítimos e utilizamos a técnica de amostragem validação cruzada com 10 repetições [22].

Os resultados dos classificadores foram avaliados nas métricas de: precisão, revocação, medida F e acurácia. Diagramas de caixa e bigode foram construídos para uma análise estatística descritiva e aplicamos o teste de hipótese ANOVA, com intervalo de confiança de 0,95 , no software RStudio, a fim de verificar se o desempenho dos algorítimos apresentava diferença estatística significativa.

\section{RESUltAdos E DisCUSSÕES}

Os resultados dos experimentos de classificação são apresentados na Tabela 1.

Tabela I

RESUlTADOS DO DESEMPENHO DOS ALGORITMOS NO PROCESSO DE CLASSIFICAÇÃO

\begin{tabular}{lccccc}
\hline Classe & Algoritmo & $\begin{array}{c}\text { Precisão } \\
\text { média (\%) }\end{array}$ & $\begin{array}{c}\text { Revocação } \\
\text { média (\%) }\end{array}$ & $\begin{array}{c}\text { Medida-F } \\
\text { média (\%) }\end{array}$ & $\begin{array}{c}\text { Acurácia* } \\
\text { (\%) }\end{array}$ \\
\hline Amendoim Bravo & & 36,8 & 20,9 & 26,7 & \\
Capim Amargoso & \multirow{2}{*}{ k-NN } & 64,2 & 50,3 & 56,4 & 86,77 \\
Milho & & 55,6 & 6,1 & 11 & \\
Fundo & & $\mathbf{8 9 , 7}$ & $\mathbf{9 6 , 3}$ & $\mathbf{9 2 , 9}$ & \\
\hline Amendoim Bravo & & 1 & 10,4 & 18,9 & \\
Capim Amargoso & Random & 87,5 & 63,0 & 73,2 & \multirow{2}{*}{89,64} \\
Milho & Forest & 1 & 3,7 & 7,1 & \\
Fundo & & $\mathbf{8 9 , 7}$ & $\mathbf{9 9 , 3}$ & $\mathbf{9 4 , 2}$ & \\
\hline Amendoim Bravo & & 63,6 & 36,6 & 46,4 & \\
Capim Amargoso & \multirow{2}{*}{ SVM } & 85,5 & 72 & 78,2 & \multirow{2}{*}{$\mathbf{9 1 , 3 4}$} \\
Milho & & 81,4 & 42,7 & 56 & \\
Fundo & & $\mathbf{9 2 , 7}$ & $\mathbf{9 7 , 7}$ & $\mathbf{9 5 , 2}$ & \\
\hline
\end{tabular}

Observamos inicialmente que o desempenho individual por classe e algoritmo (k-NN, Random Forest e SVM), são diferentes (Tabela 1), mas muito próximos em algumas classes. Contudo o algoritmo SVM, neste experimento, obteve o maior desempenho com acurácia (classificação correta) de 91,34\%.

Analisando esses resultados a partir da estatística descritiva, podemos observar na Figura 6, pelos diagramas de caixa e bigode (boxplot), que os valores das medianas dos algoritmos são diferentes, tendo o algoritmo SVM obtido o maior valor. Quanto à dispersão dos resultados, percebemos uma variação muito grande, sendo que os valores obtidos pelo algoritmo SVM variaram menos e estão na maior faixa de valores.

Na Figura 7 podemos observar que os valores das medianas e da maioria das dispersões foram diferentes, tendo como maior destaque a classe fundo com mediana próxima de $92 \%$. Quanto à dispersão dos dados, percebe-se que são assimétricas, com pouca sobreposição de valores entre as classes.

Na Tabela 2 temos o resultado do teste ANOVA que comparou o desempenho alcançado pelos algoritmos, constatandose que os valores de desempenho obtidos não apresentam diferença significativa, tendo probabilidade de se repetirem. Contudo no desempenho entre classes mas entra as classes isso não ocorre para as métricas revocação e medida F. Portanto, estas variações de desempenho podem ter ocorrido tanto pelo 


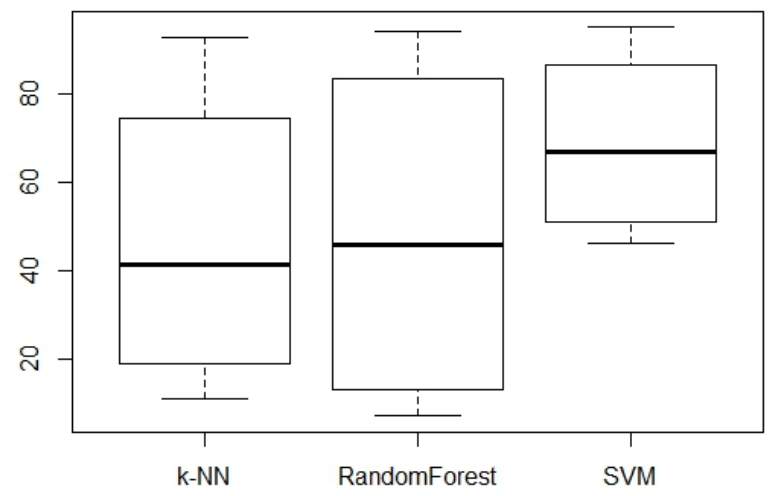

Figura 6. Diagrama de caixa e bigode comparando o desempenho entre as classes para a métrica Medida-F

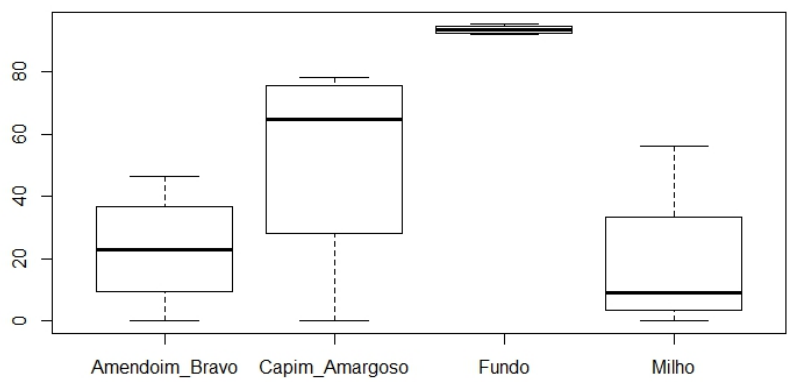

Figura 7. Diagrama de caixa e bigode comparando o desempenho entre as classes para a métrica Medida-F

acaso, como pela obtenção de uma amostra muito pequena por classe, prejudicando o desempenho dos algoritmos.

Tabela II

REsultados Do TESTE ANOVA

\begin{tabular}{lccc}
\hline Comparação & $\begin{array}{c}\text { Precisão } \\
\text { (valor-p) }\end{array}$ & $\begin{array}{c}\text { Revocação } \\
\text { (valor-p) }\end{array}$ & $\begin{array}{c}\text { Medida F } \\
\text { (valor-p) }\end{array}$ \\
Desempenho/Algoritmo & 0,1603 & 0,0683 & 0,0782 \\
Desempenho /Classe & 0,0636 & 0,0002 & 0,0013 \\
\hline
\end{tabular}

A figura 8 apresenta a matriz de confuso do algoritmo SVM tendo em vista que este obteve o maior valor absoluto de acúrcia entre os algoritmos utilizados. De acordo com a figura, a classe fundo obteve o maior número de instancias classificadas corretamente, seguida pelas classes Capim amargoso, Amendoim bravo e Milho.

$\mathrm{Na}$ figura 9 podemos observar o resultado do processo de classificação de uma nova imagem com o algoritmo SVM. Vi-

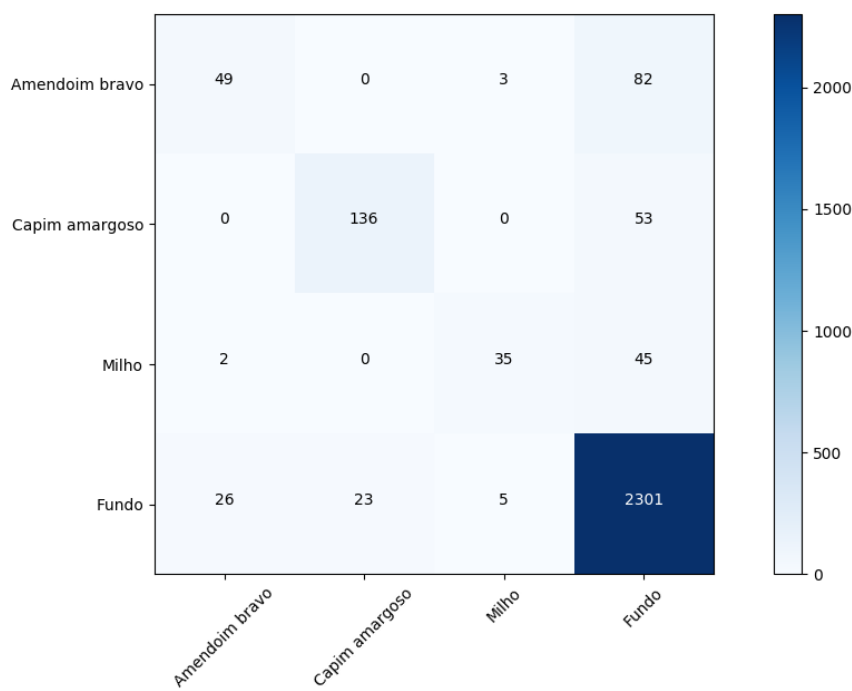

Figura 8. Matriz de confusão do algoritmo para SVM que obteve o melhor resultado entre os comparados

sualmente percebemos que plantas daninhas foram classificadas como fundo, prejudicando o desempenho de classificação correta por classe.

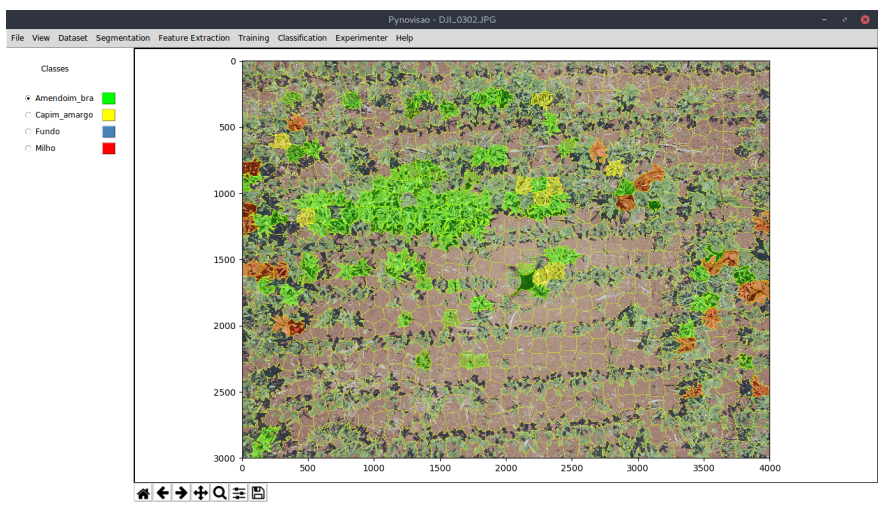

Figura 9. Detecção de plantas daninhas em uma imagem da lavoura de soja

\section{CONCLUSÃO}

Pode-se concluir que a abordagem proposta de visão computacional e imagens capturadas por VANT apresentaram bons resultados, porém na detecção de plantas daninhas em lavoras de soja não foi bom, fato este que pode ter ocorrido porque o número de imagens utilizadas no treinamentos das classes alvo foram menores se comparados com as imagens de fundo. $\mathrm{O}$ algoritmo SVM apresentou o melhor desempenho, na maioria das métricas analisadas, em comparação com os demais algoritmos testados. Para trabalhos futuros, pretendemos aumentar o banco de treinamento das classes de plantas daninhas e testar o desempenho da abordagem para diferentes alturas e com algoritmos de aprendizagem profunda. 


\section{AGRADECIMENTOS}

O presente trabalho foi realizado com apoio da Coordenação de Aperfeiçoamento de Pessoal de Nível Superior Brasil (CAPES) - Código de Financiamento 001, da NVIDIA Corporation pela doação da placa gráfica e da Fundação de Apoio ao Desenvolvimento do Ensino, Ciência e Tecnologia do Estado de Mato Grosso do Sul (FUNDECT).

VI.

\section{REFERÊNCIAS}

[1] E. Voll, D. L. P. Gazziero, A. M. Brighenti, F. S. Adegas, C. d. A Gaudêncio, and C. E. Voll, A dinâmica das plantas daninhas e práticas de manejo. Embrapa Soja Londrina, 2005.

[2] M. A. Rizzardi and N. G. Fleck, "Métodos de quantificação da cobertura foliar da infestação de plantas daninhas e da cultura da soja," Ciência rural. Santa Maria. Vol. 34, n. 1 (jan./fev. 2004), p. 13-18, 2004.

[3] T. Brosnan and D.-W. Sun, "Inspection and grading of agricultural and food products by computer vision systems-a review," Computers and electronics in agriculture, vol. 36, no. 2-3, pp. 193-213, 2002.

[4] S. Finch, A. Samuel, and G. P. Lane, Lockhart and Wiseman's Crop Husbandry Including Grassland. Elsevier, 2014.

[5] F. Garcia-Ruiz, S. Sankaran, J. M. Maja, W. S. Lee, J. Rasmussen, and R. Ehsani, "Comparison of two aerial imaging platforms for identification of huanglongbing-infected citrus trees," Computers and Electronics in Agriculture, vol. 91, pp. 106-115, 2013.

[6] J. Torres-Sánchez, F. López-Granados, A. I. De Castro, and J. M. Peña-Barragán, "Configuration and specifications of an unmanned aerial vehicle (uav) for early site specific weed management," PloS one, vol. 8 , no. 3, p. e58210, 2013.

[7] J. Torres-Sánchez, J. M. Peña, A. I. de Castro, and F. López-Granados, "Multi-temporal mapping of the vegetation fraction in early-season wheat fields using images from uav," Computers and Electronics in Agriculture, vol. 103, pp. 104-113, 2014.

[8] J. M. Peña, J. Torres-Sánchez, A. I. de Castro, M. Kelly, and F. LópezGranados, "Weed mapping in early-season maize fields using objectbased analysis of unmanned aerial vehicle (uav) images," PloS one, vol. 8, no. 10, p. e77151, 2013.

[9] M. Pérez-Ortiz, J. Peña, P. A. Gutiérrez, J. Torres-Sánchez, C. HervásMartínez, and F. López-Granados, "A semi-supervised system for weed mapping in sunflower crops using unmanned aerial vehicles and a crop row detection method," Applied Soft Computing, vol. 37, pp. 533-544, 2015.

[10] M. Pérez-Ortiz, J. M. Peña, P. A. Gutiérrez, J. Torres-Sánchez, C. Hervás-Martínez, and F. López-Granados, "Selecting patterns and features for between-and within-crop-row weed mapping using uavimagery," Expert Systems with Applications, vol. 47, pp. 85-94, 2016.

[11] F. López-Granados, J. Torres-Sánchez, A. Serrano-Pérez, A. I. de Castro, F.-J. Mesas-Carrascosa, and J.-M. Pena, "Early season weed mapping in sunflower using uav technology: variability of herbicide treatment maps against weed thresholds," Precision Agriculture, vol. 17, no. 2 , pp. $183-199,2016$.

[12] F. Castaldi, F. Pelosi, S. Pascucci, and R. Casa, "Assessing the potential of images from unmanned aerial vehicles (uav) to support herbicide patch spraying in maize," Precision Agriculture, vol. 18, no. 1, pp. 7694, 2017.

[13] J. A. Hartigan and M. A. Wong, "Algorithm as 136: A k-means clustering algorithm," Journal of the Royal Statistical Society. Series C (Applied Statistics), vol. 28, no. 1, pp. 100-108, 1979.

[14] M. J. Swain and D. H. Ballard, "Color indexing," International journal of computer vision, vol. 7, no. 1, pp. 11-32, 1991.

[15] R. M. Haralick, "Statistical and structural approaches to texture," Proceedings of the IEEE, vol. 67, no. 5, pp. 786-804, 1979.

[16] T. Ojala, M. Pietikäinen, and T. Mäenpää, "Multiresolution gray-scale and rotation invariant texture classification with local binary patterns," IEEE Trans. Pattern Anal. Mach. Intell., vol. 24, no. 7, pp. 971-987, 2002. [Online]. Available: http://dx.doi.org/10.1109/TPAMI.2002.1017623

[17] N. Dalal and B. Triggs, "Histograms of oriented gradients for human detection," in international Conference on computer vision \& Pattern Recognition (CVPR'05), vol. 1. IEEE Computer Society, 2005, pp. 886-893.
[18] M. K. Hu, "Visual Pattern Recognition by Moment Invariants," IRE Transactions on Information Theory, vol. 8, pp. 179-187, 1962.

[19] T. K. Ho, "Random decision forests," in Proceedings of 3rd international conference on document analysis and recognition, vol. 1. IEEE, 1995, pp. 278-282.

[20] N. S. Altman, "An introduction to kernel and nearest-neighbor nonparametric regression," The American Statistician, vol. 46, no. 3, pp. $175-185,1992$.

[21] J. Platt et al., "Probabilistic outputs for support vector machines and comparisons to regularized likelihood methods," Advances in large margin classifiers, vol. 10, no. 3, pp. 61-74, 1999.

[22] R. Kohavi, "A study of cross-validation and bootstrap for accuracy estimation and model selection." Lawrence Erlbaum Associates, vol. 14 p. $1137-1145,1995$. 